\title{
Multiple unit root tests under uncertainty over the initial condition: some powerful modifications
}

\author{
Christoph Hanck
}

Received: 25 November 2010 / Revised: 30 March 2011 / Published online: 22 April 2011

(C) The Author(s) 2011. This article is published with open access at Springerlink.com

\begin{abstract}
We modify the union-of-rejection unit root test of Harvey et al. "Unit Root Testing in Practice: Dealing with Uncertainty over the Trend and Initial Condition" (Harvey, Econom Theory 25:587-636, 2009). This test rejects if either of two different unit root tests rejects but controls the inherent multiple testing issue by suitably modifying the critical values to ensure the desired null rejection probability. We evaluate the new tests' power relative to existing ones' and to the Gaussian asymptotic power envelope. An empirical application illustrates the usefulness of the new statistics.
\end{abstract}

Keywords Unit root tests $\cdot$ Meta test $\cdot$ Multiple testing

JEL Classification $\quad \mathrm{C} 12 \cdot \mathrm{C} 22$

\section{Introduction}

As there is no uniformly most powerful unit root test (Elliott et al. 1996), there is and will be no consensus to always use a single test. Specifically, Müller and Elliott (2003) show the Dickey and Fuller (1979) test ( $O$ for OLS detrended) to be powerful if the initial condition of the series is large, while the GLS test of Elliott et al. (1996, $Q$ for quasi-differenced) performs well for small initial conditions. ${ }^{1}$ Since the statistics are imperfectly correlated, one test might reject the unit root null while the other does not,

\footnotetext{
1 Similar considerations arise under uncertainty over the presence of a deterministic trend. We focus on the problem of uncertainty over the initial condition for brevity, but most conclusions also apply under uncertainty over the trend.
}

C. Hanck $(\varangle)$

Department of Economics and Econometrics, Rijksuniversiteit Groningen, Nettelbosje 2,

9747 AE Groningen, The Netherlands

e-mail: c.h.hanck@rug.nl 
complicating the test decision. The 'solution' to reject if either $O$ or $Q$ reject does not yield a level- $\alpha$ test as it ignores the multiple testing nature of the problem.

Harvey et al. (2009, HLT) propose a level $-\alpha$ 'Union-of-Rejections' $(U R)$ test that rejects if either $O$ or $Q$ exceed their $\alpha$-level critical value adjusted by a suitable constant $\psi^{\alpha}$. Building on Bayer and Hanck (2009), we modify their idea by showing that power gains are possible in parts of the parameter space when scaling each critical value by separate constants $\psi_{O}^{\alpha}, \psi_{Q}^{\alpha}$. We also use Fisher's (1932) famous $P$-value combination test to derive 'meta' unit root tests.

The Fisher test is more powerful than the best of the individual tests when these have similar power, and has good power when combining more than two tests. Our modified $U R$ test is most useful when the individual tests' power differ strongly; its power is always close to that of the better individual test, and slightly higher than HLT's for moderate and large initial conditions.

Section 2 presents the model and test procedures. Section 3 provides local power results. Section 4 reports the empirical application. Section 5 summarizes and discusses possible further research.

\section{Model and combination tests}

Assumption 1 specifies the standard DGP considered in this paper.

Assumption 1 For $t=1, \ldots, T, y_{t}=\mu+u_{t}$, where $u_{t}=\rho u_{t-1}+\epsilon_{t}, t=2, \ldots, T$. $\left\{e_{t}\right\}$ is a martingale difference sequence with $\left(0, \sigma^{2}\right)$ and $\mathrm{E} e_{t}^{4}<\infty$. The error process satisfies $\epsilon_{t}=C(L) e_{t}$ where $C(L)=1+\sum_{i=1}^{\infty} C_{i} L^{i}, C(z) \neq 0$ for all $|z| \leqslant$ 1 and $\sum_{i=0}^{\infty} i\left|C_{i}\right|<\infty$. $^{2}$

We test $\mathcal{H}_{0}: \rho=1$ against $\mathcal{H}_{1}:|\rho|<1$ using the Dickey and Fuller (1979) test (denoted $\xi_{O}$ ) and the $t$-ratio of Elliott et al. (1996), denoted $\xi_{Q}$. First compute $\hat{u}_{t}=y_{t}-\bar{y}$. The $t$-ratio for $\mathcal{H}_{0}$ in the regression $\hat{u}_{t}=\rho \hat{u}_{t-1}+\sum_{p=1}^{P} v_{p} \Delta \hat{u}_{t-p}+e_{t}$ gives $\xi_{O}$. For $Q$, let $\bar{\rho}_{T}=1-7 / T, z_{1 \bar{c}}=z_{1}, y_{1 \bar{c}}=y_{1}, z_{t \bar{c}}=1-\bar{\rho}_{T}$ and $y_{t \bar{c}}=$ $y_{t}-\bar{\rho}_{T} y_{t-1}$ for $t=2, \ldots, T$. Calculate $\hat{\phi}_{\bar{c}}=\left(\sum_{t=1}^{T} z_{t \bar{c}}^{2}\right)^{-1} \sum_{t=1}^{T} z_{t \bar{c}} y_{t \bar{c}}$ and $\hat{u}^{Q}=$ $y_{t}-\hat{\phi}_{\bar{c}}$. The $t-$ ratio for $\mathcal{H}_{0}$ in $\hat{u}_{t}^{Q}=\rho \hat{u}_{t-1}^{Q}+\sum_{p=1}^{P} v_{p}^{Q} \Delta \hat{u}_{t-p}^{Q}+e_{t}^{Q}$ gives $\xi_{Q}$.

Assumption 2 controls, through $\kappa$, the magnitude of the initial condition $u_{1}=y_{1}-\mu$ under $\rho_{T}=1-c / T, c>0$ (Müller and Elliott 2003). It ensures that $u_{1}=\mathcal{O}_{p}\left(T^{1 / 2}\right.$ ) such that $u_{1}$ matters for the local distribution. The normality assumption allows to provide a power envelope, see below.

Assumption 2 For $\rho=\rho_{T}, \eta \sim \mathcal{N}\left(0, \kappa^{2}\right)$ and $\omega_{\epsilon}^{2}=\sigma^{2} C(1)^{2}, u_{1}$ is generated as $u_{1}=\eta \omega_{\epsilon}\left(1-\rho_{T}^{2}\right)^{-0.5 .3}$

The following Lemma of HLT recalls the local distribution of the $\xi_{j}$.

\footnotetext{
2 Assumption 1 rules out drift in $y_{t}$. The working paper discusses (similar) results to those below with drift in $y_{t}$.

${ }^{3}$ HLT find similar results for $u_{1}$ fixed, whence we omit a detailed discussion thereof.
} 
Lemma 1 (Harvey et al. 2009) Under Assumptions 1, 2 and $c \geqslant 0, \xi_{O} \rightarrow_{\mathrm{d}}$ $\left(K_{c}^{\mu}(1)^{2}-K_{c}^{\mu}(0)^{2}-1\right)\left(4 \int_{0}^{1} K_{c}^{\mu}(r)^{2} \mathrm{~d} r\right)^{-1 / 2}$ and $\xi_{Q} \rightarrow_{\mathrm{d}}\left(K_{c}(1)^{2}-1\right)\left(4 \int_{0}^{1} K_{c}(r)^{2}\right.$ $\mathrm{d} r)^{-1 / 2}$, where $K_{c}(r)=W(r)$ for $c=0$ and $K_{c}(r)=\kappa\left(e^{-r c}-1\right) / \sqrt{2 c}+W_{c}(r)$ for $c>0$, and $K_{c}^{\mu}(r)=K_{c}(r)-\int_{0}^{1} K_{c}(s) \mathrm{d} s, W_{c}(r)=\int_{0}^{r} e^{-(r-s) c} \mathrm{~d} W(s)$ and $W(r) a$ standard Wiener process.

Lemma 1 reveals that the $\xi_{j}$ are differentially affected by $\kappa$ under $c>0$. Hence, different tests are powerful for different $\kappa$. This is the basis of the combination tests, which aim at more robust, and possibly even more powerful, tests using $\mathcal{J}:=\{O, Q\}$. HLT's $U R$ test rejects when $\xi_{O}$ or $\xi_{Q}$ exceed adjusted critical values ensuring an overall level- $\alpha$ test. Denote test $j^{\prime}$ s level $-\alpha$ critical value by $c v_{j}^{\alpha}$. The 'naive' statistic $U R^{\mathrm{n}}:=\mathbb{I}\left\{\xi_{O}<c v_{O}^{\alpha}\right\}+\mathbb{I}\left\{\xi_{O} \geqslant c v_{O}^{\alpha}\right\} \mathbb{I}\left\{\xi_{Q}<c v_{Q}^{\alpha}\right\}$, with $\mathbb{I}$ the indicator function, rejects if $U R^{\mathrm{n}}=1$. As $U R^{\mathrm{n}}$ is oversized, ${ }^{4}$ HLT suggest to reject if $U R_{\psi}=1$, where

$$
U R_{\psi}:=\mathbb{I}\left\{\xi_{O}<\psi^{\alpha} c v_{O}^{\alpha}\right\}+\mathbb{I}\left\{\xi_{O} \geqslant \psi^{\alpha} c v_{O}^{\alpha}\right\} \mathbb{I}\left\{\xi_{Q}<\psi^{\alpha} c v_{Q}^{\alpha}\right\}
$$

and $\psi^{\alpha}$ satisfies $\operatorname{Pr}\left(\xi_{O}<\psi^{\alpha} c v_{O}^{\alpha} \cup \xi_{Q}<\psi^{\alpha} c v_{Q}^{\alpha}\right)=\alpha$. However, one need not apply $\psi^{\alpha}$ to both $c v_{j}^{\alpha}$. In fact, there is a continuum of constants $\left(\widetilde{\psi}_{O}^{\alpha}, \widetilde{\psi}_{Q}^{\alpha}\right)$ yielding level- $\alpha$ $U R$ tests. Let

$$
U R_{\psi_{\mathcal{J}}}:=\mathbb{I}\left\{\xi_{O}<\widetilde{\psi}_{O}^{\alpha} c v_{O}^{\alpha}\right\}+\mathbb{I}\left\{\xi_{O} \geqslant \widetilde{\psi}_{O}^{\alpha} c v_{O}^{\alpha}\right\} \mathbb{I}\left\{\xi_{Q}<\widetilde{\psi}_{Q}^{\alpha} c v_{Q}^{\alpha}\right\}
$$

and reject if $U R_{\psi_{\mathcal{J}}}=1$. The admissible tuples $\psi^{\alpha}$ are implicitly defined by

$$
\operatorname{Pr}\left(\xi_{O}<\psi_{O}^{\alpha} c v_{O}^{\alpha} \cup \xi_{Q}<\psi_{Q}^{\alpha} c v_{Q}^{\alpha}\right)=\alpha .
$$

For each $\psi_{O}^{\alpha}$, there is exactly one $\psi_{Q}^{\alpha}$ such that (2) holds. HLT's solution $\psi^{\alpha}$ is a special case of (2). The availability of a family of tests raises the issue of which $\psi^{\alpha}$ to use. We suggest to minimize the number of cases where, given (2), $\xi_{O}$ and $\xi_{Q}$ reject under $\mathcal{H}_{0}$, i.e. to make the tests as 'uncorrelated' as possible. Since the tests' properties under local alternatives change continuously from those under $\mathcal{H}_{0}$, a powerful test will result. Concretely, select $\psi^{\alpha}$ such that

$$
\psi_{O}^{\alpha}=\arg \min _{\widetilde{\psi}_{O}^{\alpha} \in[1, \infty)}\left\{\frac{\operatorname{Pr}\left(\xi_{O}<\widetilde{\psi}_{O}^{\alpha} c v_{O}^{\alpha} \cap \xi_{Q}<\psi_{Q}^{\alpha} c v_{Q}^{\alpha}\right)}{\min \left\{\operatorname{Pr}\left(\xi_{O}<\widetilde{\psi}_{O}^{\alpha} c v_{O}^{\alpha}\right), \operatorname{Pr}\left(\xi_{Q}<\psi_{Q}^{\alpha} c v_{Q}^{\alpha}\right)\right\}}\right\} .
$$

Table 1 reports the $\psi^{\alpha} .{ }^{5}$ We find $\left(\psi_{O}^{\alpha}+\psi_{Q}^{\alpha}\right) / 2 \approx \psi^{\alpha}$. Hence, $U R_{\psi_{\mathcal{J}}}$ 'reweighs' the $\xi_{j}$.

\footnotetext{
${ }^{4}$ Under $\mathcal{H}_{0}, \operatorname{Pr}\left(\xi_{j}<c v_{j}^{\alpha}\right)=\alpha$. The size of $U R^{\mathrm{n}}$ hence equals $\operatorname{Pr}\left(\xi_{O}<c v_{O}^{\alpha} \cup \xi_{Q}<c v_{Q}^{\alpha}\right)=\operatorname{Pr}\left(\xi_{O}<\right.$ $\left.c v_{O}^{\alpha}\right)+\operatorname{Pr}\left(\xi_{Q}<c v_{Q}^{\alpha}\right)-\operatorname{Pr}\left(\xi_{O}<c v_{O}^{\alpha} \cap \xi_{Q}<c v_{Q}^{\alpha}\right)=2 \alpha-\operatorname{Pr}\left(\xi_{O}<c v_{O}^{\alpha} \cap \xi_{Q}<c v_{Q}^{\alpha}\right) \geqslant \alpha$, since $\operatorname{Pr}\left(\xi_{O}<c v_{O}^{\alpha} \cap \xi_{Q}<c v_{Q}^{\alpha}\right) \leqslant \operatorname{Pr}\left(\xi_{j}<c v_{j}^{\alpha}\right)=\alpha$.

5 It is enough to minimize over $\psi_{O}^{\alpha}$, since $\psi_{Q}^{\alpha}$ is uniquely determined by (2). We use a two-dimensional grid search to find (3), and add an $\epsilon$ to the numerator to penalize borderline cases in which, due to simulation imprecision of $W$, the numerator would else be zero and the denominator very small, but positive.
} 
Table $1 U R$ Correction factors and critical values

\begin{tabular}{cccc}
\hline$\alpha$ & $\psi_{O}^{\alpha}$ for $\xi_{O}$ & $\psi_{Q}^{\alpha}$ for $\xi_{Q}$ & $c v_{\{O, Q\}}^{\alpha}$ for $\chi_{\mathcal{J}}^{2}$ \\
\hline 0.01 & 1.059 & 1.071 & 15.730 \\
0.05 & 1.086 & 1.110 & 10.440 \\
0.10 & 1.095 & 1.164 & 8.248 \\
\hline
\end{tabular}

Columns 2 and 3 give the constants to adjust the critical values of the statistics $\xi_{O}$ and $\xi_{Q}$ for the $U R$ test (2). The corresponding scaling factors $\psi^{\alpha}$ from HLT (see (1)) are 1.065, 1.095 and 1.126. Column 4 gives critical values of the Fisher test (4) combining $\xi_{O}$ and $\xi_{Q}$

Remark 1 Bayer and Hanck (2009) show that $U R_{\psi_{\mathcal{J}}}$ with (3) is equivalent to rejecting if the smaller $P$-value rejects when using as cutoff the level $\alpha^{\prime}<\alpha$ at which one needs to test to avoid the oversizedness of the 'naive' approach. Moreover, they show $U R_{\psi_{\mathcal{J}}}$ to outperform Bonferroni.

Another plausible aggregator for the $\xi_{j}$ is Fisher's (1932) famous test $P$-value combination statistic

$$
\chi_{\mathcal{J}}^{2}:=-2 \sum_{j \in \mathcal{J}} \ln \left(p_{j}\right)
$$

$\chi_{\mathcal{J}}^{2}$ has a well-defined asymptotic null distribution $F_{\mathcal{J}}$ as the $\xi_{j}$ converge jointly. Since the $\xi_{j}$ are nuisance parameter free and $F_{\mathcal{J}}$ takes the dependence between the $\xi_{j}$ into account, so is $F_{\mathcal{J}}$. It is straightforward to combine $|\mathcal{J}|>2$ tests, see Section 3 for results. ${ }^{6} F_{\mathcal{J}}$ is found by simulating (4). Table 1 reports critical values $c v_{\mathcal{J}}^{\alpha}$, obtained from 50,000 draws, approximating $W$ with Gaussian random walks of length $T=1,000$. As the $\xi_{j}$ correlate positively, a $c v_{\mathcal{J}}^{0.05}>9.487$, the $5 \% \chi^{2}(2|\mathcal{J}|)$ critical value that applies under independence, is necessary for a level- $\alpha$ test. $\chi_{\mathcal{J}}^{2}$ rejects whenever both $\xi_{j}$ reject, as $c v_{\mathcal{J}}^{\alpha}<-2 \sum_{j \in \mathcal{J}} \ln (\alpha)$, and is consistent, as $p_{j}=o_{p}(1)$ and thus $\chi_{\mathcal{J}}^{2} \rightarrow_{\mathrm{p}} \infty$.

\section{Asymptotic power}

We approximate rejection probabilities by simulating 100,000 replications of the distributions in Sect. 2, for $T=1,000, c \in\{0,1, \ldots, 30\}$ and $|\kappa| \in\{0,0.1, \ldots, 6\}$. Figure 1 plots power against $|\kappa|$, for $c=\{10,20\}$. (The end of sect. 3 discusses $\xi_{\hat{\rho}}$ and $\left.\chi_{\{O, Q, \hat{\rho}\}}^{2}.\right) \xi_{Q}$ is more powerful than $\xi_{O}$ for small $|\kappa|$. The power of $\xi_{O}$ increases in $|\kappa|$, that of $\xi_{Q}$ decreases. By Müller and Elliott (2003), $\kappa=1$ yields asymptotics for samples where $u_{1}$ equals one standard deviation of the unconditional distribution of $y_{t}$. Thus, $\xi_{O}$ outperforms $\xi_{Q}$ for a 'moderate' to 'large' $u_{1}$. Figure 1 also reports the Gaussian asymptotic power envelope under Assumptions 1 and 2. Specifically, Müller and Elliott (2003, p. 1274) show that the member $Q_{a}^{\mu}\left(c, \kappa^{2}\right)$ of the family $Q_{a}^{\mu}(g, k)$ is

\footnotetext{
6 This also holds for $U R_{\psi}$ from (1). One can also extend $U R_{\psi_{\mathcal{J}}}$ to $|\mathcal{J}|>2$, but it is more cumbersome to find $\psi^{\alpha}$.
} 

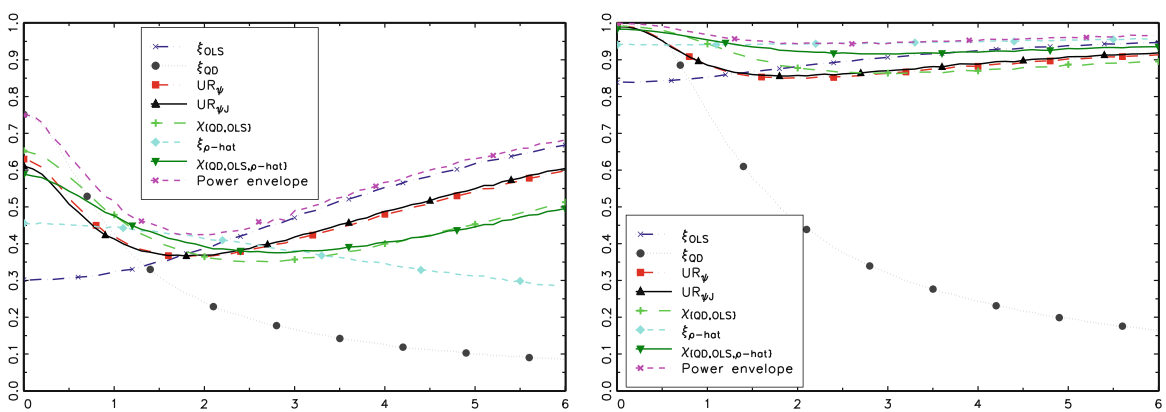

Fig. 1 Local power as a function of $|\kappa|, c=10$ and $c=20$

point optimal against an alternative having a normal $u_{1}$ with multiple $\kappa$ of the standard deviation of an $A R(1)$ process with $\rho=\rho_{T}$. Hence, $Q_{a}^{\mu}(g, k)$ traces out the power envelope over $c$ and $\kappa$.

$U R_{\psi}, U R_{\psi_{\mathcal{J}}}$ and $\chi_{\mathcal{J}}^{2}$ have power often close to the power envelope. The tests' power is always much closer to that of the better single test. Around the intersection of the individual tests, $\chi_{\mathcal{J}}^{2}$ even outperforms the individual tests. Intuitively, the $\xi_{j}$ then often just do not reject, but the joint evidence suffices. The tests are therefore "close to admissible" asymptotically (Müller 2009): Fig. 6 in HLT shows that $U R_{\psi}, U R_{\psi_{\mathcal{J}}}$ and $\chi_{\mathcal{J}}^{2}$ are never much less, and sometimes more powerful than $Q_{a}^{\mu}(10,3.8)$. Hence, there cannot be a test with much higher power for all $\kappa$.

$U R_{\psi}$ is slightly more powerful than $U R_{\psi_{\mathcal{J}}}$ for small $|\kappa|$. The ranking reverses around $|\kappa| \geqslant 0.9$, with $U R_{\psi_{\mathcal{J}}}$ outperforming $U R_{\psi}$ by up to about $1.5 \%$ (for $c=14$ and $\kappa=3.7)$. This pattern is intuitive: Table 1 shows that, relative to HLT, (3) yields a higher (lower) scaling factor for $\xi_{Q}\left(\xi_{O}\right)$. Since $\xi_{Q}$ has low power for large $|\kappa|$, little power is lost when increasing $\psi_{Q}^{\alpha}$, whereas some is gained with a lower $\psi_{O}^{\alpha}$. $\chi_{\mathcal{J}}^{2}$ outperforms $U R_{\psi_{\mathcal{J}}}$ for small-to-moderate $|\kappa| \in[0,2]$, and in particular when the individual tests have similar power. $U R_{\psi_{\mathcal{J}}}$ outperforms $\chi_{\mathcal{J}}^{2}$ when the gap between $\xi_{O}$ and $\xi_{Q}$ is large. This is intuitive as $U R_{\psi_{\mathcal{J}}}$ looks for one rejecting test, to then effectively ignore the other. $\chi_{\mathcal{J}}^{2}$ uses evidence from both tests, such that a test with low power can lead $\chi_{\mathcal{J}}^{2}$ to accept. E.g., given $P_{1}=0.03, \chi_{\mathcal{J}}^{2}$ needs $P_{2} \leqslant \mathrm{e}^{-[10.440 / 2+\ln (.03)]}=0.18$ to reject. Since no test dominates, Fig. 2 follows HLT in comparing the tests' asymptotic integrated powers over $c$, scaled relative to the power of the best test for each $|\kappa|$. The minimum integrated relative powers are highest for $U R_{\psi}, U R_{\psi_{\mathcal{J}}}$ and $\chi_{\mathcal{J}}^{2}$, suggesting robust power of combination tests.

Figure 1 also reports the power of the statistic $\xi_{\hat{\rho}}=T(\hat{\rho}-1)$ as well as of $\chi_{\{O, Q, \hat{\rho}\}}{ }^{2}{ }^{7}$ Recall $\xi_{\hat{\rho}} \rightarrow_{\mathrm{d}}\left(K_{c}^{\mu}(1)^{2}-K_{c}^{\mu}(0)^{2}-1\right) /\left(2 \int_{0}^{1} K_{c}^{\mu}(r)^{2} \mathrm{~d} r\right)$. Especially for $c=20$, adding $\xi_{\hat{\rho}}$ makes $\chi_{\mathcal{J}}^{2}$ superior to $U R_{\psi}$ and $U R_{\psi \mathcal{J}}$ : as $\xi_{\hat{\rho}}$ then has high power across

\footnotetext{
7 We also tried tests such as MSB, the weighted symmetric test (Pantula et al. 1994), R (Bhargava 1986), $\mathrm{R} / \mathrm{S}$ or the MAX-test (Leybourne 1995). However, these tests' local power is similar to that of $\xi_{Q}$, so that adding these to $\chi_{\mathcal{J}}^{2}$ does not improve the performance of $\chi_{\mathcal{J}}^{2}$. Detailed results are available.
} 
Fig. 2 Asymptotic relative integrated powers against $|\kappa|$

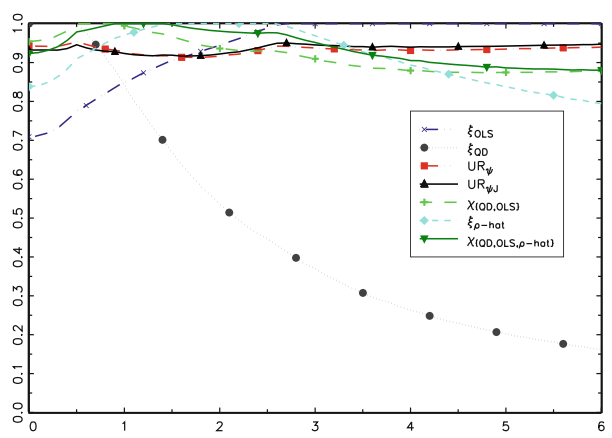

$|\kappa|, \chi_{\{O, Q, \hat{\rho}\}}^{2}$ all but avoids the power dip for intermediate $|\kappa|$ observed for $U R_{\psi}$ and $U R_{\psi_{\mathcal{J}}}$. Conversely, it is unsurprising that $U R_{\psi}$ and $U R_{\psi_{\mathcal{J}}}$ outperform $\chi_{\{O, Q, \hat{\rho}\}}^{2}$ for larger $|\kappa|$ as they are less affected by $\xi_{Q}$.

\section{Recursive testing for Purchasing Power Parity}

We study whether the tests mitigate the effect of the initial condition. Specifically, we recursively test whether the Purchasing Power Parity (PPP) relation holds between the United States and the United Kingdom. Let $p_{t}$ be the log UK price index in period $t, p_{t}^{*}$ the $\log$ price index of the US and $s_{t}$ the $\log$ nominal Pound-Dollar exchange rate. The real exchange rate is then given by

$$
r_{t}=p_{t}-p_{t}^{*}-s_{t}
$$

Tests of the PPP hypothesis are naturally formulated as unit root tests on $r_{t}$ (Rogoff 1996). In line with standard practice, a constant is used in the deterministic part. We use the annual dataset of Taylor (2002), which is useful as it covers a long period, 1892 to 1996 . We gradually increase the starting date of the test sample from $t=1892$ to 1955, yielding sample sizes between 105 and 42 .

Figure 3 plots the tests' $P$ - values, and whether $U R_{\psi_{\mathcal{J}}}$ rejects. $P$ is chosen with the MAIC (Ng and Perron 2001). As power grows in $T$, all $P$-values unsurprisingly trend upwards. $\xi_{O}$ rarely rejects, whereas $\xi_{Q}$ does. The decisions do, however, also reverse: $\xi_{O}$ rejects for samples starting during WW I, while $\xi_{Q}$ does not, plausibly a period with a large $u_{1}$. Conversely, the rejections of $\xi_{Q}$ during the calm late 19th/early 20th century is in line with the good power of $\xi_{Q}$ for small $u_{1}$.

Given only the $\xi_{j}$, one would be unsure about the properties of $r_{t}$. Except for samples starting after WW II, the combination tests typically reject. Moreover, $U R_{\psi_{\mathcal{J}}}$ and the $\chi_{\mathcal{J}}^{2}$ are less sensitive to the often arbitrary choice of starting date than the single tests, exhibiting none of the abrupt shifts e.g. during WW I. Finally, for 1933-1943, both $\chi_{\mathcal{J}}^{2}$ reject while $U R_{\psi_{\mathcal{J}}}$ does not, as one marginal single rejection and one modest acceptance suffice for a rejection for the former, but not the latter. 
Fig. $3 P$-values of US/UK real exchange rate unit root tests as a function of the starting date. Visible stretches of $U R_{\psi \mathcal{J}}$ correspond to rejection periods. The horizontal dotted line is at $\alpha=0.05$

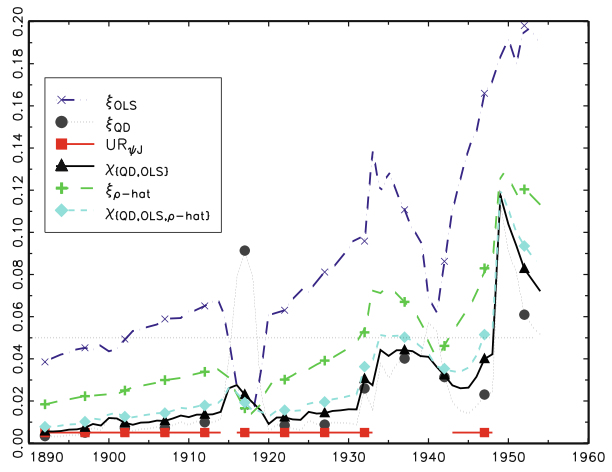

\section{Conclusion}

We propose meta tests to combine individual unit root tests. The tests take into account the multiple testing nature of running several tests and hence control size. They suitably aggregate the individual statistics or modify their critical values, based on an idea of Harvey et al. (2009). We find the tests to have good power that may be close to the Gaussian asymptotic power envelope.

These combination procedures are applicable more generally. Further work might hence develop such procedures for other problems without a uniformly most powerful test. Examples from the unit root literature include tests against nonlinear alternatives (e.g. Kruse 2011), tests which allow for general heteroscedasticity (e.g. Haldrup 1994) or panel unit root tests (e.g. Jönsson 2008).

Acknowledgements Without implicating, I would like to thank Matei Demetrescu, Graham Elliott and three anonymous referees for comments that helped to substantially improve the paper. GAUSS code used to produce all results in this paper is available upon request.

Open Access This article is distributed under the terms of the Creative Commons Attribution Noncommercial License which permits any noncommercial use, distribution, and reproduction in any medium, provided the original author(s) and source are credited.

\section{References}

Bayer C, Hanck C (2009) Combining non-cointegration tests. METEOR Res Memo 9:1-34

Bhargava A (1986) On the theory of testing for unit roots in observed time series. Rev Econ Stud 53:369-384

Dickey DA, Fuller WA (1979) Distribution of the estimators for autoregressive time series with a unit root. J Amer Statist Assoc 74:427-431

Elliott G, Rothenberg TJ, Stock JH (1996) Efficient tests for an autoregressive unit root. Ecma 64:813-836 Fisher R (1932) Statistical methods for research workers. Oliver and Boyd, London

Haldrup N (1994) Heteroscedasticity in non-stationary time series, some Monte Carlo evidence. Stat Pap 35:287-307

Harvey DI, Leybourne SJ, Taylor AMR (2009) Unit root testing in practice: dealing with uncertainty over the trend and initial condition. Econom Theory 25:587-636

Jönsson K (2008) The accuracy of normal approximation in a heterogeneous panel data unit root test. Stat Pap 49:565-579

Kruse R (2011) A new unit root test against ESTAR based on a class of modified statistics. Stat Pap 52:71-85 
Leybourne SJ (1995) Testing for unit roots using forward and reverse dickey-fuller regressions. Oxford Bull Econ Stat 57:559-571

Müller UK (2009) Comment on 'Unit root testing in practice: Dealing with uncertainty over the trend and initial condition'. Econom Theory 25:643-648

Müller UK, Elliott G (2003) Tests for unit roots and the initial condition. Ecma 71:1269-1286

$\mathrm{Ng} \mathrm{S}$, Perron P (2001) Lag length selection and the construction of unit root tests with good size and power. Ecma 69:1519-1554

Pantula SG, Gonzalez-Faria G, Fuller WA (1994) A comparison of unit-root test criteria. J Bus Econ Stat 12:449-459

Rogoff K (1996) The purchasing power parity puzzle. J Econ Lit 34:647-668

Taylor AM (2002) A century of purchasing-power parity. Rev Econ Stat 84:139-150 